\title{
Artículo Original: Lesiones Producidas por Escudos Eléctricos
}

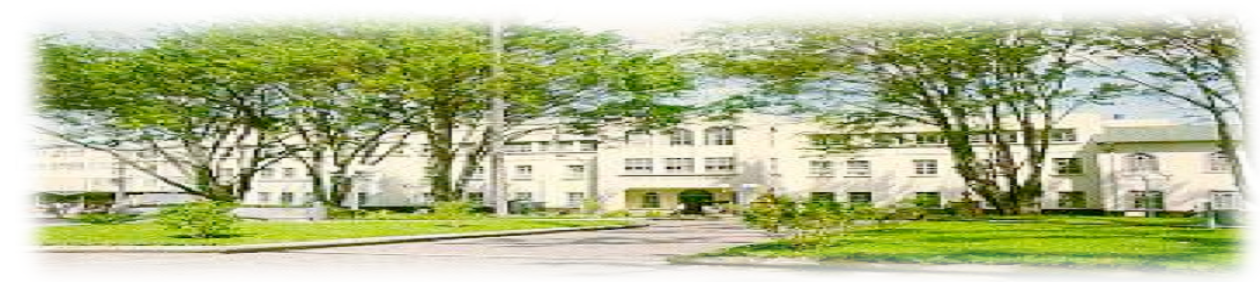

Hospital San quan de Dias. San Gosé, Costa Rica. Fundado en 1845

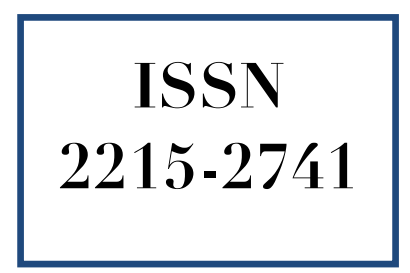

Recibido: 05/12/2015

Aceptado: 14/04/2016

Grettchen Flores Sandi ${ }^{1}$

${ }^{1}$ Médico Especialista en Medicina Legal. Magister Science en Administración de Centros y Servicios de Salud. Máster en Valoración Médica de Incapacidades y Daño Corporal. Departamento Clínico Medicina Legal, Escuela de Medicina, Universidad de Costa Rica. Correo Electrónico: grettchenflores@medicos.cr

\section{RESUMEN}

Actualmente los cuerpos policiales utilizan variedad de dispositivos de electrochoque para controlar a las personas a través de la corriente eléctrica, entre ellos los escudos eléctricos son dispositivos de contacto cuyo fin es de protección a quien los usa más que el ataque. El efecto de la exposición a fuentes externas de electricidad puede provocar lesiones al alterar el funcionamiento eléctrico normal del cuerpo humano y en caso de existir denuncias, debe comprenderse que existen factores de riesgo que constituyen múltiples variables imposibles de controlar por los usuarios de los dispositivos, al aumentar la intensidad y duración de la exposición a la corriente eléctrica: factores incontrolables, como son las lesiones provocadas por la caída al suelo de la persona y factores de riesgo potencial (cardiopatía previa, estado de intoxicación, entre otros) que pueden ser desconocidos por parte de los oficiales de policía en el momento de su utilización, indistintamente se utilice con una o varias personas, y que pueden poner en riesgo la vida de quien o quienes reciben la descarga. En el lugar de los hechos este escudo pasa a ser un indicio de origen físico que debe protegerse y levantarse adecuadamente teniendo en cuenta que puede ser determinante para el esclarecimiento del hecho, por lo que requiere una debida cadena de custodia. Sin embargo, precisamente la poca investigación sobre efectos perjudiciales a la salud por las descargas de los escudos eléctricos, hace que no existan normas de protección y seguridad en relación a su uso que deban conocer los investigadores que asisten a un lugar de los hechos donde es posible estos se encuentren, salvo las normas usuales de protección para cualquier equipo eléctrico. El análisis pericial requiere valorar la prueba con rigor crítico, para establecer y reconstruir el mecanismo y dinámica del hecho, indicando si este dispositivo fue o no utilizado, aportando elementos de prueba para la identificación del autor.

\section{PALABRAS CLAVE}

Armas eléctricas. Escudos eléctricos. Lesiones físicas. Ciencias forenses
Rev Cl EMed UCR

www.revistaclinicahsjd.ucr.ac.cr abril 2016 


\section{ABSTRACT}

Currently the police officers use a variety of electroshock devices to control people through the power supply, including electrical contact shields, devices whose purpose is to protect the user rather than the attack. The effect of exposure to external sources of electricity can cause injury by altering the normal electrical functions of the human body. Should be understood that there are risk factors, multiple variables impossible to control by users of the devices: uncontrollable factors that increase the intensity and duration of exposure to the current electric, such as injuries caused by falling of the person to the ground and potential risk factors (previous heart disease, intoxication, etc.) that may be unfamiliar by police officers when used with either with one or more people, and they can endanger the life of the person or people receiving the shock. In the scene, this shield becomes a physical clue to be protected and properly taken up considering that it may be decisive for the clarification of the issues, requiring therefor proper chain of custody. However, the very little research on harmful to health by discharges of electrical shields, causes no standards for the protection and safety in relation to its use to be known by the researchers who attend a scene where these devices are possible located, except for the standards of protection for any electrical equipment. The expert analysis requires assessing evidence with rigor criticism to establish and rebuild the mechanism and dynamics of the issue, indicating whether or not this device was used, providing evidence for the identification of the author.

\section{KEY WORDS}

Electroshock weapon. Stun shield. Physical injuries. Forensic sciences

\section{INTRODUCCIÓN}

Para las ciencias forenses, un arma es todo elemento capaz de aumentar el potencial humano para atacar o defenderse. En tal sentido, es posible distinguir el concepto de "armas propias" como todo elemento fabricado y destinado con el fin de ser un arma, es decir, cuya finalidad de su uso es simplemente ser un arma, por ejemplo, una lanza, pistola, revólver, escudo, casco, entre otros.
Es así que han sido creados diferentes tipos de armas, entre las que se encuentran las armas eléctricas que se introdujeron inicialmente como una alternativa a las armas de fuego de la policía, hechas por diferentes fabricantes basados en el mismo principio: generar impulsos breves de alto voltaje. ${ }^{1}$ Actualmente los cuerpos policiales penitenciarios y civiles utilizan una gran variedad de dispositivos de electrochoque para controlar a las personas a través de la corriente eléctrica. La tecnología incluye pistolas paralizantes (dispositivos que aplican impulsos eléctricos de alta tensión), porras eléctricas (una variante de pistolas paralizantes, que permiten a los usuarios mantener más distancia de su atacante), "tasers" (pistolas de aire para administrar choques a distancia), cinturones paralizantes (usados por los sujetos por ejemplo, en sala de juicios, capaces de ser activados de forma remota) y escudos paralizantes (diseñados como escudos policiales antidisturbios, pero con electrodos que permiten a los usuarios aplicar descargas eléctricas). ${ }^{2}$

El fin de los escudos eléctricos es de protección a quien los usa más que el ataque, porque son estructurados con policarbonato anti-rotura transparente, ofrecen completa protección al impacto de piedras o cuchillos y a la amenaza de líquido ácido o inflamable. Además, sus fabricantes mencionan otros beneficios de uso, como bajo impacto de corriente eléctrica, manija impactante fácil de operar, sustancialmente más liviano que otros tipos de escudo existentes (más de $20 \%$ más liviano), apto para usuarios diestros y zurdos (no se necesita un ajuste adicional) y los tiradores para derechos/zurdos están conectados al escudo. Sus protuberancias están diseñadas para la defensa, durabilidad del producto y resistencia, además de que pueden ser utilizados por diferentes cuerpos policiales (policía civil, fuerzas antidisturbios y policía penitenciaria) y en su diseño se puede incluir una marca privada (nombre del cuerpo policial o logotipo). En cuanto a su estructura, la longitud es $1.000 * 610 \mathrm{~mm}$ y el espesor de la pared de 3-4 mm. ${ }^{3}$

En Costa Rica, su uso reciente aunado a los riesgos conocidos para la salud y la vida que implica la exposición a otras fuentes de corriente eléctrica como ocurre en la electrocución y electrofulguración, hace necesario que el médico forense conozca las posibles lesiones y mecanismos de lesión que puede producir el uso de este tipo de dispositivos por las posibles implicaciones legales que 
puede conllevar su uso, debido a la importancia que adquiere el examen médico legal como prueba para resolver mejor ante denuncias que conozca alguna autoridad judicial, como podría ser casos de muerte o lesiones en el contexto de utilización policial o muertes en custodia.

$\mathrm{Y}$ es que en su práctica cotidiana, los médicos forenses valoran personas vivas y fallecidas con traumatismos eléctricos ocurridos tanto en los hogares y en la industria como por efectos de la electricidad atmosférica, en su valoración consideran que el impacto de la corriente eléctrica produce lesiones locales y efectos electroquímicos, térmicos y mecánicos generales que a menudo conducen a condiciones clínicas potencialmente mortales como trastornos cardíacos y respiratorios, fibrilación ventricular, shock e incluso la muerte, análisis que llega a ser trascendente dentro de un proceso judicial, La pregunta de investigación resulta ser: ¿Las personas que sufren descargas eléctricas por el uso de estos escudos podrían presentar también daños o incluso la muerte dado que el cuerpo humano normal tiene una serie de procesos físico-químicos que se afectan ante el contacto con una fuente externa de electricidad?, de lo que se deriva la hipótesis: "El uso de escudos eléctricos puede no resultar inocuo para la salud de las personas".

El objetivo de esta investigación es conocer el efecto en el cuerpo humano del uso de armas eléctricas tipo escudos, determinando las lesiones, mecanismos de lesión y posibles riesgos mortales y si existen normas de protección y seguridad en relación a su uso que deban conocer los investigadores que asisten a un lugar de los hechos.

\section{EXPOSICIÓN DE HECHOS}

En Costa Rica, el tema sobre el riesgo para la integridad física de las personas por el uso de los escudos eléctricos quedó en evidencia por hechos recientes:

En fecha 23 de julio de 2015, el Director de la Policía Penitenciaria del Ministerio de Justicia y Paz solicitó a la Escuela de Medicina de la Universidad de Costa Rica información relacionada con posibles consecuencias para la salud de las personas a causa de los escudos eléctricos. Al respecto explica que en el año 2014, el Ministerio de Justicia y Paz realizó la compra de escudos eléctricos o de electrochoque, los cuales fueron distribuidos en los distintos centros penales del país. Aclara que el tema estaba siendo analizado por distintas entidades, tales como el Mecanismo Nacional de Prevención de la Tortura, el cual es un organismo adscrito a la Defensoría de los Habitantes de la República, además de la Sala Constitucional, ante un recurso de hábeas corpus presentado por un privado de libertad. Diligencias que aún se encontraban pendientes de resolución en ese momento. El objetivo de esta solicitud era contar con criterios de seguridad de que estos dispositivos no dañan la salud o integridad de las personas privadas de libertad, ya sea a corto o largo plazo.

El 20 de agosto de 2015 se dio a conocer en los medios de comunicación costarricense la noticia de que un privado de libertad, de 30 años de edad, estando en una celda de máxima seguridad del Centro de Atención Institucional La Reforma (ubicado en San Rafael de Alajuela), sufrió quemaduras en los brazos, fractura de antebrazo izquierdo (tercio medio de la ulna que ameritó inmovilización y tratamiento antinflamatorio), además de otras lesiones, mientras se cubría la descarga eléctrica de un escudo por parte de funcionarios de la Policía Penitenciaria. Los hechos se dieron el 09 de junio de 2015, luego de que regresó de una cita médica, cuando reclamó su comida estando en una celda en el módulo Máxima E, del centro penal, caracterizado por enfrentamientos entre privados de libertad y personal de seguridad.

Precisamente, ya ese tribunal había intervenido por hechos similares en ese módulo. Tras el hecho el privado de libertad interpuso un recurso de hábeas corpus ante la Sala Constitucional contra el centro penal, el cual fue declarado con lugar por unanimidad, los siete magistrados consideraron esa medida desproporcionada e irrazonable que lesiona la integridad física del privado de libertad. $^{4-6}$ Al resolver el caso los magistrados conocieron el dictamen del Departamento de Medicina Legal que acreditó las lesiones del privado de libertad (mediante valoración efectuada el 12 de junio de 2015).

De acuerdo con la sentencia 2015-12947 de la Sala Constitucional, fechada 19 de agosto de 2015, el altercado se dio cuando este reclamó su comida y empezó a golpear el portón de su celda. Se tomó en cuenta que el privado de libertad estaba desarmado, que en la acción intervinieron va- 
rios oficiales para reducirlo a la impotencia y que fue golpeado al punto de fracturarle el antebrazo y simultáneamente se utilizó un escudo eléctrico, lo cual en el contexto citado, estimó la Sala que resulta una medida desproporcionada e irrazonable que lesiona la integridad física del privado de libertad. La sentencia agrega que esta Sala acogió una medida establecida en el nuevo protocolo de Seguridad Penitenciaria sobre el Uso de Escudos Electrónicos en Centros Penitenciarios que restringe el uso de estos dispositivos a situaciones de emergencia en que se haya ocasionado un disturbio violento colectivo y que lo prohíbe expresamente cuando se trata del abordaje de un solo privado de libertad. Este protocolo establece que por ningún motivo podrán utilizarse escudos eléctricos como una forma de maltrato, tortura, tratos crueles, inhumanos o degradantes, ya sea contra una o varias personas privadas de libertad.

Aunque no se menciona el modelo exacto del escudo en cuestión, los que se adquirieron son semejantes a los que muestra la siguiente fotografía y que pueden identificarse en videos tomados por los privados de libertad de este centro penal y puestos a disposición a través del sitio web youtube. ${ }^{7}$

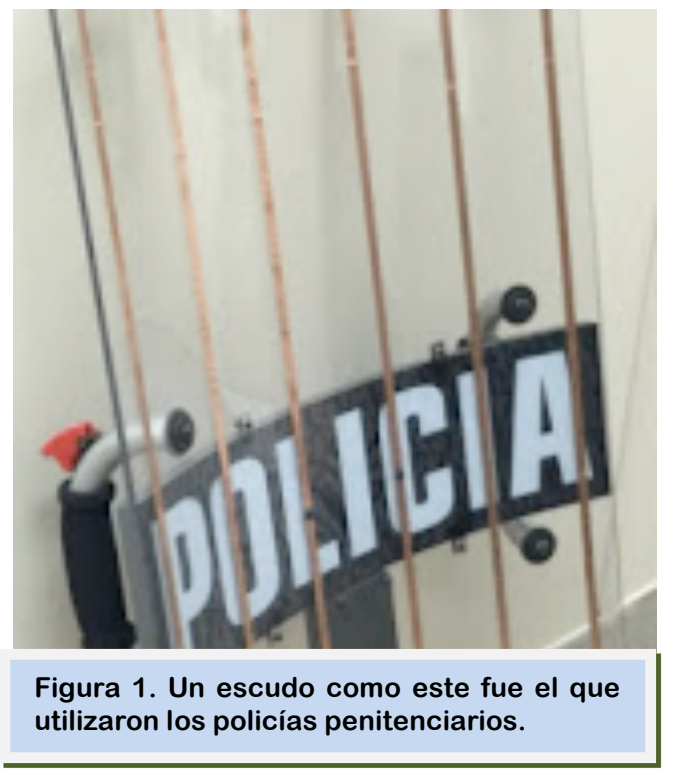

\section{ASPECTOS TÉCNICOS DE SU USO}

Como se ha mencionado, en todo organismo humano se producen corrientes eléctricas minúsculas debidas a las reacciones bioquímicas de las funciones corporales normales, es decir en el metabolismo normal se produce una reorganización de partículas cargadas, que incluso pueden ser documentadas mediante diversas pruebas médicas diagnósticas como por ejemplo los electroencefalogramas, electrocardiogramas y las técnicas de registro neurofisiológico (potenciales evocados). En este contexto resultaría lógico considerar que el efecto de la exposición a fuentes externas de electricidad como serían los escudos eléctricos, podría provocar algún tipo de daño corporal al alterar el funcionamiento eléctrico normal del cuerpo humano.

El análisis médico legal inicial que permita posteriormente un posible establecimiento de nexo de causalidad con los hallazgos clínicos encontrados en el peritaje, implica conocer aspectos técnicos de este tipo de armas. De acuerdo con Aguilar $y$ otros, $2013,^{8}$ es un concepto básico que los efectos que las armas eléctricas producen sobre el cuerpo humano dependen de las características del aparato que genera la electricidad, de la manipulación de la persona que usa el aparato y de factores internos del cuerpo al cual se aplica la electricidad, según se muestra en el Cuadro 1.

Otro aspecto importante a considerar desde el punto de vista legal, es que el permiso para usar armas de fuego no habilita para usar las eléctricas, de ahí que cualquier persona podría utilizarlas. Como se desprende de los hechos descritos, el escudo eléctrico resulta ser un dispositivo de descarga por contacto (DDC), pues para producir la descarga eléctrica sobre la persona necesita entrar en contacto directo con ella, produciendo un dolor muy intenso en la zona de contacto.

Además otro aspecto técnico a tomar en cuenta es que en el caso de las armas eléctricas, las descargas que producen son de tensión alterna caracterizada por tener valores variables de amplitud y generalmente es periódica, es decir, se repite en el tiempo, su funcionamiento se basa en el transformador eléctrico. Para considerar los efectos potenciales de las armas eléctricas la Comisión Electrotécnica Internacional emitió la norma CEI 479, en la que se habla de los efectos de la electricidad sobre el cuerpo humano, que define diversos parámetros, tales como la impedancia total del cuerpo en función de la tensión y la frecuencia aplicadas, o los efectos fisiopatológicos que puede producir una corriente 
alterna a su paso por un cuerpo humano con un recorrido de la mano izquierda a los dos pies. ${ }^{9} \mathrm{De}$ acuerdo con esta norma el efecto sobre el organismo en función del tiempo de exposición y la corriente pasa desde una zona de umbral de percepción $0,5 \mathrm{~mA}$ en corriente alterna $(2 \mathrm{~mA}$ en corriente continua) hasta el umbral de fibrilación ventricular (en corriente continua es muy superior que en corriente alterna), según se describe en la Figura 1.

\section{Cuadro 1. Elementos que influyen en el efecto de las armas eléctricas sobre el cuerpo humano}

\begin{tabular}{l|l}
\hline Elemento & Factor \\
\hline $\begin{array}{l}\text { Características del aparato que } \\
\text { genera la electricidad }\end{array}$ & $\begin{array}{l}\text {-Intensidad, tensión y frecuencia de la electricidad aplicada, y la superficie } \\
\text { de contacto. }\end{array}$ \\
\hline Persona que manipula el arma eléctrica & $\begin{array}{l}\text {-Controla la duración del paso de la corriente eléctrica (tiempo de } \\
\text { aplicación del aparato sobre el cuerpo), el recorrido de la corriente por el } \\
\text { cuerpo (lugar de aplicación) y la superficie y presión de contacto. } \\
\text {-Las zonas menos perjudiciales para aplicar los electrodos en un cuerpo } \\
\text { humano son hombros, costillas y caderas (los efectos de la electricidad son } \\
\text { menos graves cuando la corriente no pasa a través de los centros nerviosos } \\
\text { y órganos vitales ni cerca de ellos (bulbo, cerebelo, caja torácica y } \\
\text { corazón). } \\
\text {-Son áreas sensibles los ojos, las heridas, el escroto y testículos y debe } \\
\text { evitarse el contacto con las mamas y el abdomen de mujeres embarazadas. }\end{array}$ \\
\hline Factores internos del cuerpo humano & $\begin{array}{l}\text {-Impedancia del cuerpo, su temperatura, grado de humedad de la piel, } \\
\text { grosor de la epidermis, etc. }\end{array}$ \\
\hline
\end{tabular}

Fuente: Elaboración propia a partir de fuentes consultadas.

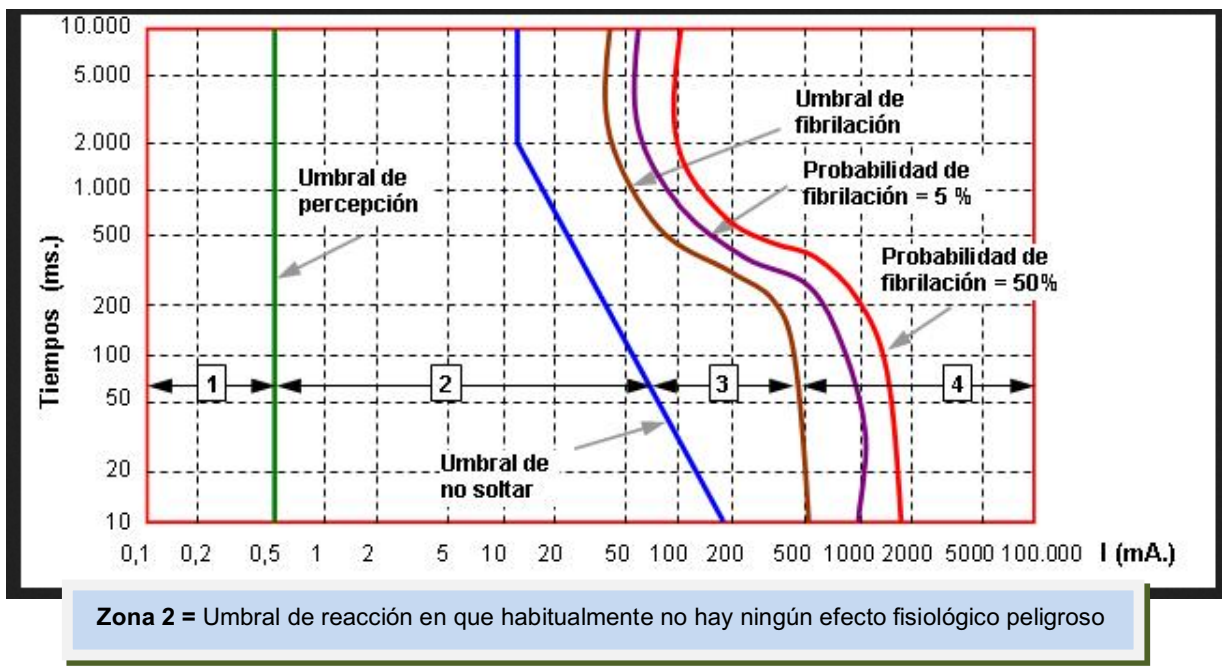

Figura 1. Efectos de la corriente alterna $(15-100 \mathrm{~Hz})$ sobre las personas en función del tiempo de exposición y la corriente. 
Estos efectos ocurren porque una vez efectuada la descarga, el impulso eléctrico no se localiza únicamente entre los electrodos, sino que pasa por las zonas de menor resistencia neurovasculares, la superposición de la frecuencia al ritmo nervioso y circulatorio puede llegar a producir una alteración que se traduce en espasmos, sacudidas y fibrilación ventricular. Por otra parte para los efectos sobre la piel, la norma CEI 479 establece unas curvas que indican las alteraciones de la piel humana en función de la densidad de la corriente que circula por un área determinada $(\mathrm{mA} / \mathrm{mm} 2) \mathrm{y}$ el tiempo de exposición a esa corriente, que van desde la ausencia de lesiones hasta la carbonización.

De lo que anterior se desprende, que sí son posibles efectos a nivel local y sistémico en el cuerpo de una persona expuesta a armas eléctricas, aunque existan estudios que concluyan que su uso sí es seguro, ya que se debe tener presente el sesgo importante en la valoración de la seguridad recogida en las referencias bibliográficas, así como el conflicto de intereses, ya que quienes concluyen que estas armas son seguras son autores relacionados con las casas fabricantes, pues muchos de los estudios han sido en parte financiados por quienes producen los dispositivos. ${ }^{10-11}$ No obstante, a partir de estudios realizados con dispositivos eléctricos similares, se ha sugerido que las personas pueden tener un riesgo de salud aumentado que los hace más vulnerables a presentar complicaciones en su salud, que van más allá del efecto temporal reportado por el fabricante. ${ }^{12-14}$ Además se han realizado estudios en animales con la dificultad de extrapolación de resultados a casos en humanos. Sin embargo, no hay estudios que correlacionen el efecto de muerte con la edad, el sexo, el peso, el índice de masa corporal ni la utilización de fármacos de quienes reciben la descarga, por lo que se ha concluido que la necesidad de valorar múltiples factores dificulta establecer una verdadera relación causal entre la exposición y efectos a la salud. ${ }^{8,15}$

Por otra parte, se han realizado estudios en personas sanas con una sola aplicación, menor o igual a 5 segundos de duración, lo cual no representa eventos de uso legal de fuerza, situaciones extremas en que se reciben múltiples descargas. Además los oficiales deben considerar que su uso no implica del todo ausencia de riesgo por las posibles caídas o traumas que puede sufrir la víctima al recibir la descarga. ${ }^{16-18}$ En cambio, el impacto en personas en que existen condiciones médicas ha recibido menos atención, y la evidencia reportada es escasa, sí existen casos aislados como el ocurrido en diciembre de 1995, en que el oficial penitenciario Harry Landis del Departamento de Justicia Criminal de Texas, con aparente historia de problemas cardíacos fue reportado muerto después de recibir dos descargas de 45000 voltios de un escudo eléctrico, como parte de un ejercicio de entrenamiento, en que la causa de muerte (disrritmia cardíaca debido a bloqueo coronario) se analizó considerando que la muerte no hubiera ocurrido sino hubiese recibido estas descargas. $^{2,19}$

En todo caso, en la atención de emergencias de personas que han recibido descargas de estos dispositivos se indica una evaluación que comprenda causas traumáticas, médicas y toxicológicas de los síntomas que atribuyen a la corriente eléctrica. ${ }^{20}$ El cuadro 2 esquematiza los efectos que se han descrito por el uso de este tipo de armas, no obstante la mayoría de la información disponible no se refiere a los escudos eléctricos, sino a otros dispositivos electrónicos similares que emiten descargas eléctricas.
Rev Cl EMed UCR

$\underline{\text { www.revistaclinicahsjd.ucr.ac.cr }}$

abril 2016 
Cuadro 2: Lesiones descritas por uso de armas eléctricas

\begin{tabular}{|c|c|c|}
\hline Nivel & Sitio & Efecto \\
\hline \multirow[t]{2}{*}{ Local } & Tejido Cutáneo & $\begin{array}{l}\text { - lesiones pares (cuando son consecuencia de la acción de los electrodos), } \\
\text { maculares eritematosas, redondeadas, de entre } 2 \text { y } 5 \mathrm{~mm} \text {, bien circunscritas, con } \\
\text { puntura central, separadas unos } 5 \mathrm{~cm} \text {, que pueden persistir hasta } 2 \text { horas. } \\
\text { - en caso de que no haya penetrado la punta, se encontrarán mínimas quemaduras } \\
\text { en la zona de aplicación. Histológicamente se describe presencia de elongación } \\
\text { nuclear de las células epidérmicas, homogeneización eosinófila del tejido } \\
\text { subepidérmico e infiltración neutrófila precoz. } \\
\text { - con una intensidad elevada y cuando las superficies de contacto son } \\
\text { importantes se puede llegar a la fibrilación ventricular sin ninguna alteración de } \\
\text { la piel. }\end{array}$ \\
\hline & Tejido muscular & $\begin{array}{l}\text {-tetanización muscular con ruptura tendinosa } \\
\text {-no hay estudios concluyentes sobre el incremento de la creatina quinasa liberada } \\
\text { como consecuencia de daño mecánico o metabólico al músculo (rabdomiólisis } \\
\text { puede ser consecuencia de la actividad muscular intensa, compresión sobre el } \\
\text { músculo, fenómenos de hipertermia, tóxicos musculares directos o bien por la } \\
\text { lesión eléctrica producida por este tipo de armas). }\end{array}$ \\
\hline \multirow[t]{3}{*}{ Sistémico } & Cardiovasculares & $\begin{array}{l}\text { - no hay estudios concluyentes sobre fibrilación ventricular. } \\
\text { - posible mecanismo de captura miocárdica en los dispositivos intracardíacos. }\end{array}$ \\
\hline & Endocrinas & $\begin{array}{l}\text { - activación de la respuesta al estrés humano: descarga catecolaminérgica } \\
\text { (efectos cardiovasculares, respiratorios, musculares, hiperkalemia, acidosis, } \\
\text { hiperglucemia y elevación del hematócrito), } \\
\text { - no se ha detectado hipertermia. } \\
\text {-debate en torno a muerte súbita (especialmente en individuos bajo efectos de } \\
\text { ciertas drogas, alcohol, o que presentan ciertas condiciones que comprometan su } \\
\text { salud). }\end{array}$ \\
\hline & Otras & -catarata, aborto, daño cerebral \\
\hline Indirectas & $\begin{array}{l}\text { Traumatismos (caídas, } \\
\text { heridas, etc) }\end{array}$ & $\begin{array}{l}\text { - heridas, contusiones, fracturas, lesiones tendinosas, trauma cráneo encefálico. } \\
\text { - existen reportes de perforación ocular, desprendimiento de retina, perforación } \\
\text { de la faringe, neumotórax, compresión y fractura de la médula espinal y ataques } \\
\text { epilépticos cuando el dispositivo eléctrico es aplicado en la parte superior del } \\
\text { tórax o directamente en la cabeza de un individuo. }\end{array}$ \\
\hline
\end{tabular}

Fuente: Elaboración propia a partir de fuentes consultadas.

\section{OBSERVACIONES DEL CASO}

En relación al caso descrito, se tiene que de acuerdo con las especificaciones del Director de la Policía Penitenciaria del Ministerio de Justicia y Paz, los escudos adquiridos que son los que están en discusión cumplen con las siguientes especificaciones:

Norma IEEE STD 80, clausula 6 y 5.3

Voltaje: < $50000 \mathrm{~V}$

Tensión producida por el equipo: 900 a $1200 \mathrm{~V}$

Intensidad eléctrica: $20 \mathrm{~mA}$ (0.020 A)

En el análisis causal de cualquiera de las lesiones observadas, debe considerarse que aunque la fuente es una batería de tensión continua, el equipo la modifica para crear una fuente alterna, lo que hace disminuir los rangos entre los que oscilan las zonas de umbral de percepción (zona 1) y de umbral de fibrilación ventricular (zona 4), variando los posibles efectos fisiológicos sobre el organismo en función del tiempo de exposición y la corriente. Considerando la intensidad eléctrica especificada de $20 \mathrm{~mA}$ (0.020 amperios) sí es posible se produzcan efectos fisiológicos en una persona expuesta dependiendo del tiempo de exposición.

Aunque no se específica la duración del paso de la corriente, de acuerdo con las especificaciones: según la norma IEEE STD 80, ${ }^{21}$ clausula 6 y 5.3, en estudios hechos con animales de un peso corporal y cardíaco semejante al humano el corazón aumenta la susceptibilidad a la 
fibrilación ventricular cuando el tiempo de exposición a la corriente es aproximado al período de latido cardíaco y el riesgo disminuye si el tiempo de exposición se encuentra en el rango de 0.06 a $0.3 \mathrm{~s}$ (60 a $300 \mathrm{~ms})$. Tomando en consideraición la Figura 1, no se producirían efectos fisiológicos peligrosos a exposiciones de 60 a $300 \mathrm{~ms}$.

Además, la víctima por sí misma tendería a alejarse de la fuente de energía lo que haría disminuir el tiempo de exposición. Sí debe tenerse en cuenta, como se ha mencionado, que en la actualidad no existen investigaciones concluyentes sobre el modo en que el uso de estos dispositivos (escudos eléctricos específicamente) afecta a las personas con problemas de salud subyacentes (cardiópatas, epilépticos) o que estén bajo los efectos de ciertos medicamentos, drogas o alcohol en el momento de ser expuestos a la descarga eléctrica y que esta descarga puede agravar un problema de salud preexistente en quien la recibe. Por lo que acreditar la seguridad de estos dispositivos para la salud e integridad de las personas privadas de libertad a corto o largo plazo, debe considerar no solo la escasa información de medicina basada en evidencia, sino que el estado de salud, el padecimiento de ciertas enfermedades o condiciones hereditarias, el uso de medicamentos, el abuso de drogas o del alcohol, suponen estados físiológicos que representan variables imposible de controlar por los oficiales de policía. Además, la persona a quien se aplica la descarga eléctrica con este dispositivo aún en condición de salud óptima puede sufrir algún tipo de traumatismo al momento de recibir la descarga.

\section{CONCLUSIONES}

Tal y como se ha mencionado sí es posible se presenten efectos fisiológicos y médicos en las personas que reciben descargas de armas eléctricas. Sin embargo, precisamente la poca investigación sobre efectos perjudiciales a la salud por las descargas de los escudos eléctricos, hace que no existan normas de protección y seguridad en relación a su uso que deban conocer los investigadores que asisten a un lugar de los hechos donde es posible estos se encuentren, salvo las normas usuales de protección para cualquier equipo eléctrico (no exponerse a las descargas, evitar daños en el equipo por golpes, derrame de residuos entre las partes y la protección adecuada del equipo que evite su manipulación, desarme, deterioro o una mala utilización que no sea la especificada).

Es posible concluir que en la investigación de hechos de este tipo, debe comprenderse que existen factores de riesgo que constituyen múltiples variables imposibles de controlar por los usuarios de los dispositivos que sí pueden poner en riesgo la vida, al aumentar la intensidad y duración de la exposición a la corriente eléctrica: factores incontrolables, como son las lesiones provocadas por la caída al suelo de la persona y factores de riesgo potencial (cardiopatía previa, estado de intoxicación, entre otros) que pueden ser desconocidos por parte de los oficiales de policía en el momento de su utilización indistintamente se utilice con una o varias personas, y que pueden poner en peligro la vida de quien o quienes reciben la descarga. De tal forma que siempre que se cuestione o no la relación causal entre una posible lesión y los hechos denunciados -en este caso la descarga eléctrica de alto voltaje y bajo amperaje- en una investigación de un hecho penalmente punible, se requiere una investigación de manera técnicacientífica, que permita obtener elementos de prueba necesarios para reconstruir lo sucedido y determinar de manera fehaciente el rol y grado de participación de esta descarga eléctrica en el resultado dañoso, así como la identidad de la persona que utilizó el dispositivo, en lo cual el principio de intercambio de Locard aporta elementos para relacionar o descartar a un presunto responsable.

Luego de la delimitación y preservación del lugar del hecho, la observación y el registro del lugar: en el lugar del hecho este escudo pasa a ser un indicio de origen físico que debe protegerse y levantarse adecuadamente teniendo en cuenta que puede ser determinante para el esclarecimiento del hecho, siendo importantes su recolección y envío al laboratorio de ciencias forenses, bajo la debida cadena de custodia que se puede desarrollar en cuatro pasos:

-Paso 1. Escena de los hechos: Aquí se inicia la recolección de indicios orgánicos e inorgánicos, se hace el levantamiento del escudo (se recupera individualmente), luego de haber sido fijado fotográficamente, se hace uso de las normas de seguridad (levantándolo con las manos con guantes descartables y equipo de bioseguridad para no contaminarlo en caso de que tenga restos

Rev Cl EMed UCR www.revistaclinicahsjd.ucr.ac.cr abril 2016 
de algún otro indicio en su superficie como sangre o huellas) se registra en una planilla, con una descripción del mismo y las circunstancias del levantamiento. Se identifica al funcionario que lo recolectó. Se describe las condiciones de preservación (envuelto en papel y debidamente protegido como indicio, debe estar debidamente rotulado y numerado).

-Paso 2. Traslado: Trasladado a los laboratorios forenses. Este traslado se realiza bajo custodia de policía judicial, debe quedar registrado también, detallando en la planilla fecha, hora, nombre y firma de quién la recibe y quién la entrega, entre otros datos.

-Paso 3. Análisis: El escudo llega a los laboratorios forenses, en caso de que el escudo presente a su vez otro tipo de indicios: uno inorgánico (huellas papilares) y otro orgánico (presunto tejido hemático), antes de enviar a pericias físicas, primero se realizaría el levantamiento de la muestra biológica para determinación de especie, grupo sanguíneo y factor Rh, y eventual cotejo de $\mathrm{ADN}$, teniendo cuidado de no frotar ni limpiar la parte del escudo en que pueden descansar las huellas, e indicando en la solicitud al laboratorio que se debe primero hacer el análisis biológico y después el dactiloscópico (sino se indica es posible que se invierta el orden o se quede sin realizar el levantamiento de rastros papilares), para luego solicitar el análisis del escudo al área o sección de pericias físicas (debe incluir sus características considerar el tipo de corriente eléctrica, intensidad eléctrica, voltaje y duración de las descargas, así como considerar técnicas de laboratorio que permitan determinar si se ha utilizado o no un arma de estas características, mediante la detección de elementos metálicos en piel de la víctima procedentes de los electrodos). La recepción en cada una de estas secciones también debe ser registrada con los datos correspondientes, así como cada movimiento del escudo dentro de las instalaciones y cada lugar de permanencia y personal a cargo de cada fase del análisis.

-Paso 4. Almacenamiento: Finalmente, es almacenado en un lugar como una bóveda o anaquel donde pueda mantenerse correctamente sin contaminarse. El funcionario que lo recibe para almacenaje también lleva una planilla de cadena de custodia, registrando su nombre y firma $\mathrm{y}$ datos relativos a su recepción $\mathrm{y}$ almacenamiento.

Esto basado en los principios probatorios de protección de la prueba, legalidad, veracidad y comprobación, garantizando la identidad del indicio (escudo) y los resultados del estudio.

Además es posible que el perito médico a quien le corresponda posteriormente la valoración de un denunciante, en su investigación considere importante para su pericia observar el escenario para determinar las circunstancias que rodearon el hecho, y que en su análisis considere el informe de pericias físicas elaborado sobre las características del arma eléctrica tipo escudo que haya sido utilizado. Incluso las marcas patrón que puede dejar el escudo en la piel de la víctima deben ser consideradas por el perito médico y lesiones concomitantes (en caso de autopsia también se deben considerar lesiones concomitantes vitales o postmórtem).

Si bien en el caso analizado no se realizó un peritaje sobre el escudo utilizado, ni se logró establecer la identidad de los oficiales que aplicaron la descarga eléctrica, una correcta valoración del escudo como elemento indiciario exige un estudio en el lugar donde se haya realizado el hecho punible.

En síntesis, el análisis pericial requiere valorar la prueba con rigor crítico, para establecer y reconstruir el mecanismo y dinámica del hecho, indicando si este dispositivo fue o no utilizado, aportando elementos de prueba para la identificación del autor.

\section{BIBLIOGRAFÍA}

1. Laima S Fomin D Jasulaitis A Andriuškevičiūtė G Chmieliauskas S Sabaliauskas V y otro. The effect of conducted electrical weapons on the human body. Acta Med Litu 2014; 21: 73-80.

2. Committee on Corrections. Conditions of Confinement on the Use of Stun Shields by de New York City Department of Correction. The Record 2001; 56(4): 536-561. 
3. TAR Ideal Concepts Ltd. Escudo eléctrico para disturbios. Detalle del producto. [Internet]. Israel: TAR Ideal Concepts Military and police equipment \& training - one stop shop. [citado 4 Ago 2015]. Disponible en: http:/www.tarideal.com/esp/Soluciones /AGENTES_POLICIALES/ESCUDOS/ TA03050/ESCUDO_ELECTRICO_PA RA_DISTURBIOS

4. Madrigal C. Sala Constitucional prohíbe el uso de escudos eléctricos que causaron quemaduras a reo. [Internet]. Costa Rica: laprensalibre.cr. [actualizado 20 Ago 2015; citado 18 Set 2015]. Disponible en: http:/www.prensalibre.cr/Noticias/detal le/37246/361/sala-constitucionalprohibe-el-uso-de-escudos-electricosque-causaron-quemaduras-a-reo

5. Miranda H. Preso en La Reforma sufrió quemaduras. Sala condena uso de escudo eléctrico contra un presidiario. Resolución no prohibe que se emplee en casos graves como motines. [Internet]. Costa Rica: La Nación. [actualizado 21 Ago 2015; citado 18 Set 2015]. Disponible en: http://www.nacion.com/sucesos/poderjudicial/Sala-condena-escudo-electricoreo_0_1507249294.html

6. Rojas P. En pleito por comida, reo sufre golpiza y descarga eléctrica en La Reforma. [Internet]. Costa Rica: crhoy.com NOTICIAS 24/7. [actualizado 20 Ago 2015; citado 18 Set 2015]. Disponible en:

http://www.crhoy.com/en-pleito-porcomida-reo-sufre-golpiza-y-descargaelectrica-en-la-reforma/

7. Reos denuncian uso de escudos eléctricos en la reforma. [Internet]. Costa $\mathrm{Ri}$ ca: Extra Tv. [actualizado 4 Sep 2015; citado 16 Oct 2015]. Disponible en: www. youtube.com/watch?v=VFA6CRT 6wAg
8. Aguilar-Pallarès A Morante-Barragán JF Novelle-Rodríguez M SubiranaDomènech M. Armas eléctricas: ¿qué sabemos? ¿qué ignoramos?. Cuad Med Forense 2013;19(3-4):75-86

9. Pérez L (Redactor). NTP 400: Corriente eléctrica: Efectos al atravesar el organismo humano. [Internet]. España: Instituto Nacional de Seguridad e Higiene en el Trabajo; 1995. [citado 26 Nov 2015]. Disponible en: http://www.insht.es/InshtWeb/Contenid os/Documentacion/FichasTecnicas/NTP /Ficheros/301a400/ntp_400.pdf

10. Azadani PN Tseng ZH Ermakov S Marcus GM Lee BK. Funding source and author affiliation in TASER research are strongly associated with a conclusion of device safety. Am Heart J 2011; 162 (3):533-537

11. Kroll MW Lakkireddy DR Stone JR Luceri RM. TASER Electronic Control Devices and Cardiac Arrests: Coincidental or Causal. Circulation 2014; 129:93-100

12. The Expert Panel on the Medical and Physiological Impacts of Conducted Energy Weapons. The Health Effects of Conducted Energy Weapons. [Internet]. Ottawa, Canada: Council of Canadian Academies \& Canadian Academy of Health Sciences; 2013. [citado 6 Oct 2015]. Disponible en: http://scienceadvice.ca/uploads/eng/asse ssments\%20and\%20publications\%20an $\mathrm{d} \% 20$ news\%20releases/cew/cew_fullre porten.pdf

13. Kunz SN Grove N Fischer F. Acute pathophysiological influences of conducted electrical weapons in humans: $A$ 
review of current literature. Forensic Sci Int 2012; 221:1-4.

14. O'Brien AJ Thom K. Police use of TASER devices in mental health emergencies: A review. Int J Law Psychiatry 2014; 37: 420-426

15. Jauchem JR. Exposures to Conducted Electrical Weapons (Including TASER ${ }^{\circledR}$ Devices): How Many and for How Long are Acceptable? J Forensic Sci 2015; 60: S116-S129.

16. AELE Law Enforcement Legal Center. Taser ${ }^{\circledR}$ Electronic Control Devices (ECDs): An "Intermediate" Use of Force? AELE Mo L J 2010; (2):101109.

17. Hi JD Miner JR Lakireddy DR Bultman LL Heegaard WG. Cardiovascular and Physiologic Effects of Conducted Electrical Weapon Discharge in Resting Adults. Acad Emerg Med 2006; 13:589595

18. Hudak LA Marti JJ Houry DE. Bilateral patellar tendon rupture after stun gun shock. Injury 2011; 42: 186-188

19. New York Times News Service. Jolt from electronic riot shield fatal for state prison worker. [Internet]. USA: The Victoria Advocate. [actualizado 2 Dec 1995; citado 26 Nov 2015]. Disponible en:

https://news.google.com/newspapers?ni $\mathrm{d}=861 \&$ dat $=19951202 \& \mathrm{id}=\mathrm{mVFSAAA}$ AI-

BAJ\&sjid $=8$ jUNAAAAIBAJ\&pg $=2035$ ,6160495\&hl=en

20. Bozeman WP Hauda II WD Heck JJ, Graham DD Martin BP Winslow JE. Safety and Injury Profile of Conducted
Electrical Weapons Use by Law Enforcement Officers against Criminal Suspects. Ann Emerg Med 2009; 53(4): 480-489.

21. Substations Committee of the IEEE Power Engineering Society. IEEE Guide for Safety in AC Substation Grounding. IEEE Std 80-2000. [Internet]. New York, USA: Institute of Electrical and Electronics Engineers, Inc; 2000. [citado 4 Ago 2015]. Disponible en: http://www.dee.ufrj.br/ acsl/grad/equip amentos/IEEE-std80.pdf

\section{AGRADECIMIENTOS}

Al Dr. Alfredo López Dávila, profesor del Departamento de Fisiología de la Escuela de Medicina de la Universidad de Costa Rica por su aporte bibliográfico y a Dra. Nora Viviana Sotelo y Lic, Javier Pachamé profesores de Ciencias Forenses de la Facultad de Ciencias Médicas de Universidad Nacional de La Plata (Argentina) por la lectura del borrador de este artículo. 